\title{
Blast resistance in rice: a review of conventional breeding to molecular approaches
}

\begin{abstract}
Blast disease caused by the fungal pathogen Magnaporthe oryzae is the most severe diseases of rice. Using classical plant breeding techniques, breeders have developed a number of blast resistant cultivars adapted to different rice growing regions worldwide. However, the rice industry remains threatened by blast disease due to the instability of blast fungus. Recent advances in rice genomics provide additional tools for plant breeders to improve rice production systems that would be environmentally friendly. This article outlines the application of conventional breeding, tissue culture and DNA-based markers that are used for accelerating the development of blast resistant rice cultivars. The best way for controlling the disease is to incorporate both qualitative and quantitative genes in resistant variety. Through conventional and molecular breeding many blast-resistant varieties have been developed. Conventional breeding for disease resistance is tedious, time consuming and mostly dependent on environment as compare to molecular breeding particularly marker assisted selection, which is easier, highly efficient and precise. For effective management of blast disease, breeding work should be focused on utilizing the broad spectrum of resistance genes and pyramiding genes and quantitative trait loci. Marker assisted selection provides potential solution to some of the problems that conventional breeding cannot resolve. In recent years, blast resistant genes have introgressed into Luhui 17, G46B, Zhenshan 97B, Jin 23B, CO39, IR50, Pusa1602 and Pusa1603 lines through marker assisted selection. Introduction of exotic genes for resistance induced the occurrence of new races of blast fungus, therefore breeding work should be concentrated in local resistance genes. This review focuses on the conventional breeding to the latest molecular progress in blast disease resistance in rice. This update information will be helpful guidance for rice breeders to develop durable blast resistant rice variety through marker assisted selection.
\end{abstract}

Keyword: Blast disease; Conventional breeding; Molecular approaches; Tissue culture 